\title{
Apuntes para la transversalización de la perspectiva de género. La revisión de propuestas pedagógicas de cuatro asignaturas de la UNLP
}

\author{
Notes for mainstreaming the gender perspective. The review of \\ pedagogical proposals of four subjects of the UNLP
}

\section{María Laura Viscardi}

https://orcid.org/0000-0003-2964-0637

mlviscardi@gmail.com

Facultad de Trabajo Social | Universidad Nacional de La Plata | Argentina

Aimé Lescano

https://orcid.org/0000-0001-9045-0977

aimelescano@gmail.com

LabHiPsi - Laboratorio de Historia de la

Psicologia | Universidad Nacional

de La Plata | Argentina

\author{
Lucía Matteucci \\ https://orcid.org/0000-0003-4610-1176 \\ luchamate@gmail.com \\ Universidad Nacional de La Plata \\ Argentina
}

\section{RESUMEN}

Presentamos una propuesta de innovación curricular ligada a la transversalización de la perspectiva de géneros y disidencias en las propuestas pedagógicas de cuatro asignaturas pertenecientes a diferentes carreras dictadas en la Universidad Nacional de La Plata. El objetivo principal es el diseño de estrategias y herramientas que aporten a la revisión de dichas propuestas pedagógicas desde la perspectiva de géneros y disidencias, siendo los equipos docentes de las asignaturas, les destinataries directos del proyecto. Para su implementación se contempla un programa estructurado en nudos problemáticos vinculados a la revisión crítica de los contenidos y las bibliografías de los programas a partir de la identificación de sesgos de géneros y disidencias. Se focaliza en la evaluación entendida desde una mirada crítica que acompaña todo el proceso. Se destaca la necesidad de generar antecedentes en torno a un posicionamiento docente crítico acerca de la labor de formar profesionales comprometides con valores de igualdad y equidad.

\section{PALABRAS CLAVE}

perspectiva de géneros $y$ disidencias,

transversalización de género educación superior evaluación. 
KEY WORDS

gender and dissidence perspective,

gender mainstreaming,

higher education,

evaluation.

\section{ABSTRACT}

We introduce a proposal for curricular innovation linked to the mainstreaming of the gender and dissidence perspective in the pedagogical proposals of four subjects belonging to different careers taught at the National University of La Plata. The main objective of the proposal is the design of strategies and tools that contribute to the review of pedagogical proposals from the perspective of gender and dissidence, in which the direct beneficiaries of the project are the teachers of these subjects. For its implementation, a program structured in problematic nodes linked to the critical review of the contents and bibliographies of the programs is contemplated from the identification of gender biases and dissidences. It focuses on the evaluation understood from a critical perspective that accompanies the entire process. The need to generate a background around a critical teaching position about the work of training professionals committed to values of equality and equity is highlighted. 


\section{PRESENTACIÓN}

El presente trabajo es resultado de la elaboración conjunta de cuatro docentes de diferentes unidades académicas de la UNLP, y tiene el objetivo de presentar un proyecto de innovación curricular destinado al abordaje de la transversalización de la perspectiva de género en las currículas de asignaturas de cuatro facultades en las que nos desempeñamos como docentes: "Psicología II" (FPsi), "Educación para la Salud" (FCM), "Introducción a la Geografía" (FaHCE) y Teoría del Estado (FTS). Dicho proyecto fue elaborado en el marco de la Especialización en Docencia Universitaria de la Universidad Nacional de La Plata.

La enseñanza en la universidad constituye una práctica social históricamente situada en la que se ponen en juego un conjunto de mediaciones orientadas a imprimir de modo explícito o implícito un tipo de racionalidad en las prácticas que acontecen en las instituciones educativas. Enseñar entonces no se liga a la asimilación de ideas y al establecimiento de conclusiones con respecto al conocimiento público, sino que se liga al valor del conocimiento para que pueda ser reconstruido, resignificado y en que ayude a reformular las comprensiones subjetivas en las relaciones del sujeto con el mundo y consigo mismo (Edelstein, 2014: 21).

Partiendo de una perspectiva crítica, pensamos a la innovación como una ruptura, es decir como la posibilidad de interrumpir, -o romper- una determinada forma de comportamiento que se repite en el tiempo Lucarelli (2004). A su vez, así entendida, la innovación, se legitima dialécticamente con la posibilidad de relacionar esta nueva práctica con las ya existentes a través de mecanismos de oposición, diferenciación o articulación. En palabras de la autora "encuadrar de esta manera la innovación supone utilizar, a la vez la categoría de mul- 
tidimensionalidad, lo que deriva en el reconocimiento de las múltiples variables que afectan la práctica" (Lucarelli, 2004: 3).

Por otro lado, Zabalza Beraza y Zabalza Cerdeiriña (2012) sostienen que las innovaciones desbordan inmediatamente el ámbito de lo individual, o lo grupal en este caso, y se proyectan en el contexto de la institución educativa en cuestión. En este sentido, esta propuesta de innovación curricular representa un andamiaje general que requiere ser resituada en cada una de las unidades académicas en las cuales las autoras de este trabajo nos desempeñamos como docentes. Si bien la presentación representa un proyecto común, sabemos que la realidad y la cultura institucional de cada unidad académica es diferente, como distinto es también el posicionamiento, el recorrido, la apertura y sensibilidad de cada una de ellas a la importancia de la perspectiva de género en la problematización de la realidad y la formación de futures profesionales.

El marco conceptual que fundamenta esta propuesta considera al concepto de género y diversidad sexo-genérica como una de sus categorías centrales. Sintéticamente, entendemos a estas categorías en términos de construcciones sociales entramadas en relaciones de poder (Lagarde, 1996). La perspectiva de género se basa en un conjunto de teorizaciones desarrolladas en torno al género como categoría de análisis crítico y se apoya en una matriz socio histórica, dialéctica y opuesta al unicausalismo, que supera el binarismo y la heteronormatividad obligatoria. A su vez, se trata de una perspectiva que es tanto política como práctica en tanto busca no solo comprender sino accionar propuestas organizativas y de transformación de prácticas, representaciones y discursos ahistóricos, biologicistas, patriarcales, heteronormados y cisexistas.

De modo esquemático, esta propuesta se fundamenta en antecedentes relevantes a nivel nacional ligados a la transversalización de género en la currícula universitaria, tales como la experiencia de la Facultad de Ciencia Política y Relaciones Internacionales de la Universidad Nacional de Rosario, que desde 2018 viene trabajando en ese sentido (Fabbri, Moltoni, Rovetto y Ridruejo, 2020); el Programa Integral de Políticas de Género (PIPG), que funciona desde el año 2017 en la Universidad Nacional de Mar del Plata; las políticas de sensibilización en género y diversidad implementadas desde el año 2018 en el ingreso universitario en la UNLP, entre otros. En este último caso, desde ese ciclo lectivo se ha incorporado un espacio introductorio de formación en género que se articula a través de talleres específicos coordinados por la Prosecretaría de Derechos Humanos de la UNLP e implementados por la Dirección de Género, Diversidad y Derechos Humanos, donde se destaca la multidimensionalidad de la inclusión de la perspectiva de géneros, diversidades y feminismo en el ámbito de la universidad pública. 
Los programas de las asignaturas condensan y visibilizan aquello que se pretende enseñar y los modos para lograrlo. En tal sentido, toman decisiones respecto a los enfoques que son considerados relevantes o no, así como también respecto a qué actualizaciones temáticas o bibliográficas se incorporan o no. Como corolario, ejercen influencia tanto por aquello que enseñan como por aquello que no enseñan, es decir, el currículum nulo o ausente, ya sea de modo explícito o implícito, pero siempre con repercusiones prácticas (Attardo et.al., 2020).

En consecuencia, los programas de las asignaturas, sus contenidos, enfoques metodológicos, regímenes de evaluación y promoción y material bibliográfico, no son neutrales al género; entendemos que si no se atiende de modo reflexivo al trabajo con cada una de estas dimensiones curriculares, se participa en la reproducción de desigualdades estructurales. En este caso nos preguntamos por la inclusión de la perspectiva de género en los currículums de los espacios donde desarrollamos nuestra práctica docente y es desde nuestra cotidianeidad que nos interesa problematizar y pensar acciones innovadoras que nos permitan aportar a las propuestas pedagógicas y apostar a una construcción de conocimientos más igualitaria en términos de género.

En relación con el objetivo de transversalizar la perspectiva de género y disidencias, cabe destacar que por temas transversales entendemos que son aquellos que atraviesan todo el currículo, tanto de forma horizontal como vertical (Álvarez, Balaguer y Carol, 2000; citado en Díaz Barriga, 2015).

En relación con el objetivo de transversalizar la perspectiva de género y disidencias, cabe destacar que por temas transversales entendemos que son aquellos que atraviesan todo el currículo, tanto de forma horizontal como vertical (Álvarez, Balaguer y Carol, 2000; citado en Díaz Barriga, 2015). Se trata de temas que requieren un tratamiento desde una perspectiva tanto cognitiva como actitudinal. El interjuego entre estos elementos, cognoscitivos y actitudinales, le da un sentido particular a su tratamiento, puesto que no es suficiente reconocer o entender los contenidos en su nivel cognoscitivo (pero si es necesario), sino que es necesario reconocer cómo estos contenidos cognoscitivos se plasman en las instituciones y en las prácticas educativas (cómo por ejemplo la idea de que las relaciones entre los géneros involucran relaciones de poder se halla ligada a quienes efectivamente son voces autorizadas para enunciar determinados conceptos, o adquirir visibilidad como autores o autoras en un contexto histórico determinado). 
En este marco proponemos propiciar la transversalización de la perspectiva de géneros y disidencias en las propuestas pedagógicas de las materias en las que ejercemos la docencia en pos de favorecer la construcción de saberes más igualitarios.

En este marco proponemos propiciar la transversalización de la perspectiva de géneros y disidencias en las propuestas pedagógicas de las materias en las que ejercemos la docencia en pos de favorecer la construcción de saberes más igualitarios. Para ello, nos planteamos como objetivos; en primer término, revisar los programas de las asignaturas "Psicología II" (FPsi), "Educación para la Salud" (FCM), "Introducción a la Geografía" (FaHCE) y Teoría del Estado (FTS) desde la perspectiva de géneros y disidencias teniendo en cuenta dos ejes fundamentales: contenidos y bibliografías; en segundo lugar, identificar en relación con los contenidos de las asignaturas la presencia de sesgos de géneros y disidencias; en tercer lugar, revisar la bibliografía vigente de los programas de las asignaturas con perspectiva de género; y por último, y fundamentado en los objetivos anteriores, desarrollar una guía de lineamientos para identificar las dimensiones primordiales para la transversalización de la perspectiva de géneros y disidencias en las currículas. De este modo, considerando central que todas las profesiones incorporen una perspectiva crítica respecto a las desigualdades sociales por razones de género y disidencias, buscamos cuestionar la hegemonía de la heteronormatividad y el patriarcado en el ámbito académico y generar cambios en el diseño de las materias en las que somos docentes

Retomando la clasificación de campos de conformación estructural curricular establecida por Alicia de Alba (1995), nuestra propuesta aporta al campo crítico-social. Los contenidos se estructuran contemplando cuatro "nudos problemáticos" detallados a continuación:

Nudo Problemático 1: ¿Hay autoras o disidencias en tu programa?”. Nudo Problemático 2: "La lengua degenerada".

Nudo Problemático 3: "Situando al género".

Nudo Problemático 4: "Transversalizando la perspectiva de género".

\section{¿Se incluyen en las referencias bibliográficas y lecturas pro- puestas en los programas de las asignaturas varones, mujeres, personas pertenecientes al colectivo LGTTBIQ+?}

El primer nudo problemático, incorpora como temas centrales la revisión 
incluyen en las referencias bibliográficas y lecturas propuestas en los programas de las asignaturas varones, mujeres, personas pertenecientes al colectivo LGTTBIQ+? Este nudo problemático implica por lo tanto la profundización sobre la relevancia de las identidades de género y orientaciones sexuales de las autorías incluidas en los programas de las asignaturas, entendiendo que la reflexión sobre este tópico permite reflexionar de modo crítico qué voces son visibilizadas en las áreas disciplinares incluidas en los programas de las asignaturas y qué voces son invisibilizadas o sujetas a prácticas de borramiento. Por último, en términos conceptuales, implica un trabajo que discurre desde la comprensión del género como una categoría analítica relevante, a la comprensión de la violencia epistémica como práctica de exclusión sistemática de otras voces y autorías en la producción, circulación de conocimiento y en su uso pedagógico en el marco de una propuesta programática. Respecto al segundo nudo problemático, este implica atender al foco analítico representado por la dimensión del lenguaje utilizado en los programas de las asignaturas: ¿Es el lenguaje neutral al género? Entendiendo que no lo es, la revisión del modo por el cual nos referimos a les autores, las categorías y nuestres alumnes, profundizando sobre las potencialidades del uso del lenguaje no sexista o inclusivo, permite pensar otros modos de materializar las propuestas pedagógico-didácticas, en programas que sean sensibles al género. Por su parte, el tercer nudo requiere trabajar sobre la presencia y/o ausencia de las perspectivas y nociones aportadas por los estudios de género para abordar el campo problemático propuesto en la materia seleccionada. Implica discriminar este análisis atendiendo tanto a los ejes del programa, como a sus objetos de estudio, y a los contenidos mínimos de las asignaturas. De esta manera, se pretende interrogar en torno a la inclusión de la perspectiva de género y sexualidades, considerando las diferencias y desigualdades por razones sexo-genéricas en el abordaje de los contenidos, y las estrategias posibles por medio de las cuales podría incorporarse dicho abordaje. Por último, el cuarto nudo problemático, implica considerar que en definitiva la transversalización de la perspectiva de género conlleva interrogar y cuestionar todas las dimensiones que configuran el currículum: tanto las propuestas metodológicas, como las modalidades de enseñanza, como así también repensar las dinámicas de trabajo áulico propuestas, los recursos utilizados y las formas por las cuales se evalúan y acredi$\tan$ los contenidos.

Por último, el cuarto nudo problemático, implica considerar que en definitiva la transversalización de la perspectiva de género conlleva interrogar y cuestionar todas las dimensiones que configuran el currículum: tanto las propuestas metodológicas, como las modalidades de enseñanza, como así también repensar las dinámicas de trabajo áulico propuestas, los recursos utilizados y las formas por las cuales se evalúan y acreditan los contenidos. 
Concebimos la evaluación como un proceso crítico que acompaña todo el proyecto y permite recabar información para la toma de decisiones y la evaluación del proyecto, y se aborda en un doble sentido: en relación con los aprendizajes y en relación a la evaluación del proyecto de innovación (Davini, 2008). La Tabla 1, sintetiza los tipos de evaluación que contempla esta propuesta y su implementación.

Tabla 1. La evaluación del proyecto

\begin{tabular}{|c|c|}
\hline Objeto evaluado & $\begin{array}{l}\text { Las propuestas pedagógicas de las materias "Psicología II” (FPsi), “Educación para la Salud” (FCM), } \\
\text { "Introducción a la Geografía” (FaHCE) y Teoría del Estado (FTS) }\end{array}$ \\
\hline \multirow{3}{*}{ Quiénes evalúan } & $\begin{array}{l}\text { Evaluación Mixta } \\
\text { - Equipo de Cátedra } \\
\text { - Equipo de implementación del proyecto de innovación }\end{array}$ \\
\hline & $\begin{array}{l}\text { Evaluación Diagnóstica } \\
\text { - Identificación de las perspectivas de género y disidencias que les docentes sostienen } \\
\text { - Valoración de los intereses y potencialidades del equipo docente } \\
\text { - Indagación de los conocimientos previos en torno al género, la perspectiva de género y su alcance } \\
\text { en las instituciones académicas } \\
\text { - Revisión de los programas de las asignaturas puntualizando los contenidos, la bibliografía utilizada } \\
\text { y les autores propuestos. }\end{array}$ \\
\hline & $\begin{array}{l}\text { Evaluación Formativa } \\
\text { - Interpretación de avances y retrocesos en el proceso. } \\
\text { - Identificación de obstáculos en el desarrollo del proyecto. } \\
\text { - Revisión de posicionamientos y referencias teóricas en relación a la perspectiva de géneros y } \\
\text { disidencias. } \\
\text { - Favorecimiento retroalimentaciones en la marcha del proceso fundamentalmente en torno al } \\
\text { currículum y la incorporación de la perspectiva de género } \\
\text { - Reorientación de las actividades programadas. }\end{array}$ \\
\hline \multirow{3}{*}{ Momentos } & $\begin{array}{l}\text { Ex ante: Se orienta a evaluar las generalidades y la coherencia interna del proyecto a través de: } \\
\text { - Análisis de los fundamentos del proyecto, selección y organización de los contenidos, y del desa- } \\
\text { rrollo del proyecto. } \\
\text { - Análisis de la factibilidad de implementación de la propuesta. }\end{array}$ \\
\hline & $\begin{array}{l}\text { Durante: Se realiza a los fines de ir evaluando la implementación del proyecto de innovación y la } \\
\text { valoración de la apropiación del proyecto por parte de las cátedras. Para ello se propone: } \\
\text { - Realización por parte de cada cátedra de un portafolio que incorpore los materiales, discusiones y } \\
\text { avances en el proceso de revisión de los programas de las asignaturas. } \\
\text { - Realización de talleres para socializar facilitadores y obstáculos en la implementación del proyecto. }\end{array}$ \\
\hline & $\begin{array}{l}\text { Ex post: Con la intención de poder evaluar y visualizar el impacto de transversalización de la pers- } \\
\text { pectiva de género pueden considerarse las siguientes acciones: } \\
\text { - Identificación la construcción de nuevas propuestas pedagógicas con los avances realizados que } \\
\text { transversalizan la perspectiva de género y disidencias } \\
\text { - Realización de encuentros para revisar el proceso de implementación del proyecto de innovación. } \\
\text { - Presentación de informe acerca del impacto de la implementación del proyecto de innovación. }\end{array}$ \\
\hline
\end{tabular}




\section{REFLEXIONES FINALES}

Presentamos una propuesta que parte del interés por aportar a las discusiones y debates que ponen en tensión los saberes dominantes y de reproducción de conceptos y categorías en relación a la perspectiva de género en cada uno de los campos disciplinares donde ejercemos la docencia. Para ello, destacamos la relevancia de comprender la perspectiva de géneros y disidencias en las instancias de formación de grado, considerando los antecedentes actuales ligados al trabajo institucional respecto al abordaje de las violencias por razones de género.

Asumiendo la importancia de que las iniciativas innovadoras estén vinculadas al proyecto formativo global de la institución educativa, consideramos que entre los aspectos de cada cultura institucional, las trayectorias de conformación e institucionalización de cada carrera van a configurar un aspecto fundamental en la posibilidad o no de que este proyecto de innovación sea posible. Entendiendo que el recorrido desde los inicios hasta la realidad actual de los proyectos formativos en los que nos inscribimos hacen a esa historia institucional que las define y las identifica, con acuerdos, sentido común y lenguaje propio. Partiendo de dicha realidad, la propuesta busca aportar a la discusión y cuestionar la hegemonía de la heteronormatividad y el patriarcado en el ámbito académico, apostando a la generación de cambios en las asignaturas en las que nos desempeñamos como docentes, en pos de problematizar la formación de futures profesionales.

\section{Partiendo de dicha realidad, la propuesta busca aportar a la discusión y cuestionar la hegemonía de la heteronormatividad y el patriarcado en el ámbito académico, apostando a la gene- ración de cambios en las asignaturas en las que nos desempe- ñamos como docentes, en pos de problematizar la formación de futures profesionales.}

Uno de los aspectos más importantes del proyecto lo constituye la instancia de evaluación, la cual entendemos como un proceso que se sitúa en distintos momentos: antes, durante y después. Planteamos, en una primera instancia, evaluar las generalidades y la coherencia interna del proyecto a través del análisis de sus fundamentos, selección y organización de los contenidos, del desarrollo del proyecto y del análisis de la factibilidad de implementación de la propuesta. Un segundo momento se ubica durante la implementación del proyecto, donde proponemos la realización de un portafolio por cátedra que incorpore materiales y avances del trabajo de cada una de ellas. Y por último, la evaluación ex post, donde incluimos la posibilidad de poder identificar el impacto sobre los participantes de la innovación, medir los cambios reales que la propuesta provocó en las actividades educativas, 
como así también el impacto sobre las instituciones.

Destacamos la necesidad de trabajar de modo colectivo entre las diversas unidades académicas en la elaboración de antecedentes que permitan pensar la problemática de la transversalización de la perspectiva de géneros y disidencias incorporada a la labor docente comprometida con una formación profesional fundamentada en los valores de igualdad, equidad y derechos humanos en la UNLP. 


\section{BIBLIOGRAFÍA}

Attardo, C. et al. (2020). La perspectiva de género en la universidad: ¿qué enseñás y cómo lo hacés?. En C. Attardo et.al (Comp.). Apuntes sobre género en curriculas e investigación (pp. 17-36). Editorial de la Universidad Nacional de Rosario. Recuperado de http://ruge.cin.edu.ar/attachments/article/24/04714_02\%20-\%20Apuntes\%20sobre\%20g\%C3\%A9nero\%20en\%20 curr\%C3\%ADculas\%20e\%20investigaci\%C3\%B3n.pdf

Davini, M. C. (2008). Métodos de enseñanza: didáctica general para maestros y profesores. Santillana.

De Alba, A. (1995). Currículum: Crisis, Mito y Perspectiva. Miño y Dávila Editores.

Díaz Barriga, A. (2015). Curriculum: entre utopía y realidad. Amorrortu.

Edelstein, G. (2014). Una interpelación necesaria: Enseñanza y condiciones del trabajo docente en la universidad. Revista Política universitaria, 1, 20-25. Recuperado de https://iec.conadu.org.ar/files/publicaciones/1548861356_politica-universitaria-1-2014.pdf

Fabbri, L., Moltoni, R., Rovetto, F. y Ridruejo, A. (2020). De la introducción a la transversalización. Experiencias, trayectorias y estrategias desde la FCPolit a la UNR, En C. Attardo et.al (Comp.). Apuntes sobre género en curriculas e investigación (pp. 37-74). Editorial de la Universidad Nacional de Rosario. Recuperado de http://ruge.cin.edu.ar/attachments/article/24/04714_02\%20-\%20Apuntes\%20sobre\%20g\%C3\%A9nero\%20en\%20curr\%C3\%ADculas\%20e\%20investigaci\%C3\%B3n.pdf

Fernández Lamarra et. al. (2015). Reflexiones en torno al concepto de innovación y la universidad. En N. Fernández Lamarra (Comp.). La innovación en las Universidades Nacionales. Aspectos endógenos que inciden en su surgimiento y desarrollo (pp. 25-42). Universidad Nacional de Tres de Febrero.

Lagarde, M. (1996). Género y feminismo. Desarrollo humano y democracia. Editorial horas y HORAS.

Lucarelli, E. (2004). La innovación en la enseñanza ¿caminos posibles hacia la transformación de la enseñanza en la universidad? [ponencia]. $3^{\circ}$ Jornada de innovación Pedagógica en el Aula Universitaria. Universidad Nacional del Sur. Junio de 2004.

Maffia, D. (2020). Feminismo y epistemología: un itinerario político personal. En D. Maffía, A. Moreno Sardá, Y. Espinosa Miñoso, y B. Radi. Apuntes epistemológicos. (pp. 17-30). Editorial de la Universidad Nacional de Rosario. Recupe- 
rado de http://ruge.cin.edu.ar/attachments/article/24/9ee8a_01-Apuntes\%20 Epistemol\%C3\%B3gicos.pdf

Zabalza Beraza, M. y Zabalza Cerdeiriña, A. (2012). Innovación y cambio en las instituciones educativas. Homo Sapiens Ediciones. 